\title{
A pedigree-based analysis of mitochondrial DNA diversity in a dog population on the example of German Hovawarts
}

\author{
S. Zielińska ${ }^{1}$ and I. Głażewska ${ }^{2}$ \\ ${ }^{1}$ Department of Molecular Biology, University of Gdańsk, Gdańsk, Poland \\ ${ }^{2}$ Department of Plant Taxonomy and Nature Conservation, University of Gdańsk, Gdańsk, Poland
}

Correspondence to: I. Głażewska (i.glazewska@ug.edu.pl)

Received: 14 November 2014 - Revised: 12 July 2015 - Accepted: 13 July 2015 - Published: 13 August 2015

\begin{abstract}
The purpose of the article is to illustrate the use of pedigree analysis to evaluate mtDNA diversity in a selected population of pedigree dogs, to describe the paths of mtDNA inheritance and to estimate the spread of potential pedigree errors or mutations that occurred in different generations of ancestors. Hovawart, old German breed, was used as an example. The number and frequencies of mtDNA haplotypes were calculated based on numbers of dam lines and their representatives. The scale of potential errors in calculations that can result from pedigree errors or from new mutations in ancestors from the 5th or 10th ancestral generation was evaluated. The analysis included 368 breeding bitches from four German kennel organizations. The bitches represented three dam lines, with the Ho1, Ho2 and HoU mtDNA haplotypes. Significant differences in the frequency of the haplotypes in the population, from 0.27 to $73.37 \%$, and among kennel organizations and regions of the country were recorded. Considerable differences in the scale of potential errors in calculations arising from mtDNA mutations or pedigree errors were noted between 0.27 and $28.69 \%$, depending on the number of representatives of the subline in which the error appeared and the generation taken into account in the simulations. The study revealed an interesting paradox: although the differences between the haplotypes are the result of events (mutations) from thousands of years ago, the number and the frequencies of the haplotypes in the population are the result of the modern history of the population and current breeding policy.
\end{abstract}

\section{Introduction}

In recent years, genetic diversity in dogs and health issues resulting from breeding policy have met with growing interest from researchers and breeders, and diversity of mitochondrial DNA is one of the issues discussed. In a review article, Verscheure et al. (2013) cited 58 articles presenting phylogenetic and forensic mtDNA analyses published between 1995 and 2013. In the majority of the articles, mtDNA study was based exclusively on laboratory analysis of samples taken at random, and only in some were pedigree data used to select animals for study. However, the scope of pedigree information use in mtDNA studies may be considerably wider. In the analyses of Polish Hounds and Hovawart dogs (Głażewska and Prusak, 2012; Głażewska et al., 2012), pedigree data were used also to evaluate haplotype frequencies.
The starting point for these projects was the statement that since mtDNA is inherited exclusively through dam lines, the number of dam lines and their representatives correspond to the number and frequencies of mtDNA haplotypes in a population. Obviously, the necessary conditions for such studies are credible pedigrees and the lack of mutations (Głażewska et al., 2013) because they lead to incorrect calculation results. The question remains, however, of how significant the resulting inaccuracies are for calculation precision and how often they occur in a population. As a rule, the error frequency resulting from mutations is determined by the mutation rate of a given mtDNA region that is estimated on a scale of thousands of years, and, thus, significantly exceeds the period described in the pedigrees of modern breeds of domestic animals. Hence, heteroplasmy or new mutations are only occasionally reported in dog or horse studies (Klütsch et al., 
2011; Bower et al., 2013). This leads to the conclusion that the anticipated number of errors of this kind in mtDNA studies based on pedigree data should be small.

In the case of pedigree errors, they derived from the quality of a breeders work and the specificity of breeding. In light of the unmeasurable nature of these factors, it is impossible to determine a priori the quality of a given pedigree and whether the scale of errors in a pedigree database renders the results of pedigree analysis completely worthless or only lowers its accuracy without prejudicing general conclusions. Undoubtedly, more errors resulting from the incorrect identification of animals can be expected in stock animals than in those kept in separate stalls or kept singly by one owner, like dogs.

The purpose of the study was to present the possibilities offered by the use of pedigree information in mtDNA studies of dogs. The first goal was to evaluate the level of mtDNA diversity in a population, i.e. a number and frequencies of haplotypes, on the basis of the numbers of dam lines and their representatives. The second goal was to describe the ways of mtDNA inheritance and to estimate the spread of potential mutations or pedigree errors in the population. This is a very interesting topic since genealogical lines in dogs, unlike in horses, are not of interest to breeders; therefore, the line structure of dog populations is poorly described. Additionally, this knowledge can be important both theoretically and practically, in genetic, forensic and veterinary studies. The third goal of the study was to evaluate the magnitude of a potential error in the calculations, which could result from an error in a pedigree of a particular ancestor or from a new mutation in this ancestor. This allows us to determine the degree of reliability of mtDNA studies based solely on the pedigree data analysis.

German population of Hovawart dogs, used as the reference population in this study, is an excellent object for pedigree-based mtDNA analysis. The Hovawart is an old German breed with a modern history dating back to the 1920 s, and is presently bred in many countries. An extensive pedigree database on Hovawarts is publicly available on the working-dog.eu website (www.working-dog.eu). Basic information about mtDNA diversity in the breed is already known from the study performed on Polish Hovawarts (Głażewska et al., 2012), in which two mtDNA haplotypes: Hol and Ho2 were identified. The Ho2 haplotype, with the frequency in the population of approximately $58 \%$, strongly dominant $(91 \%)$ in bitches imported to Poland, which raised the question of whether this domination resulted from the dominance of $\mathrm{Ho} 2$ in the whole breed. An interesting aspect is also the number of haplotypes in Hovawarts in Germany, the breed's country of origin, and therefore being the population of a potentially greater mtDNA diversity than those found in descendant populations (Savolainen et al., 2002).

\section{Material and methods}

The analysis included 368 breeding bitches from kennels belonging to the German breeding organizations Rassezuchtverein für Hovawart-Hunde (RZV) (226 females), Hovawart Zuchtgemeinschaft Deutschland (HZD) (90 females), and Hovawart-Club (HC) (13 females) associated in the Verband für das Deutsche Hundewesen (VDH), and from kennels belonging to Spezialzuchtgemeinschaft der Hovawartzüchter Mitteldeutschlands (SZG) (39 females). The list of the bitches was compiled using data provided on the websites (www.hovawart.org; http://www.hovawarte.com; www.hovawart-club.de; www. szg-hovawart.de) of these organizations in the spring of 2013. The affiliation of each bitch to a dam line was determined with pedigree data from the working-dog database (www.working-dog.eu), which was created by Hovawart breeders and owners from many countries. In the absence of pedigree data in the working-dog database, missing information were supplemented based on data drawn from kennel websites. Four bitches, pedigree data of which were not found in the web sources, were not included to the analysis. Therefore, the analysis covered $98.9 \%$ of all breeding bitches registered in Germany in the period analysed.

On the basis of collected data, the general scheme of family relations in dam lines was composed with the use of Microsoft Visio program, and then the percentage of representatives of each dam line in the analysed group of the breeding bitches and in the different kennel organizations were calculated using Microsoft Office Excel 2007. The number of dam lines and proportion of their representatives in the population was assumed to be equal to the number and frequency of mtDNA haplotypes present in the population. Next, it was assumed that the German representatives of two dam lines, described in the study of Polish Hovawarts, have the same mtDNA haplotypes as those identified in the Polish representatives of these lines (Głażewska et al., 2012). In that study, the Ho1 and Ho2 haplotypes, GenBank accession nos. HM007196 and HM007197, were found in lines established in the 1920s by the bitches Dina (Geisler) and Dina (Brüser), respectively. The haplotypes differ by 18 nucleotides and belong to two distinct haplogroups that correspond to clad A (Ho1) and clad B (Ho2) as determined by Savolainen et al. (2002). It was also assumed that haplotypes of representatives of any other previously untested lines would be designated in the present study with the letters HoU (unknown).

The second step of the study addressed issues regarding the spread in the modern population of potential pedigree errors, leading to an incorrect affiliation of an individual to the dam line, or of mtDNA mutations, fixed and passed to subsequent generations. For the purpose of theoretical simulation, it was assumed that a pedigree error or a point mutation (referred to as EM) occurred in a given female ancestor in the 5th or 10th generation of the line. Based on the general scheme of dam lines, the founders of sublines started 
in these generations were identified, and then the percentage of descendants of these founders in the group of the bitches analysed were calculated. The numbers obtained are equal to the frequency of each of the potential EMs in the modern population.

\section{Results}

The 368 bitches analysed originated from 319 litters and were owned by 334 breeders. Only $38.32 \%$ bitches were born in the same kennel as their mothers, and $19.02 \%$ of them in the same kennel as their grandmothers. The bitches represented three dam lines that descend from Dina (Geisler), born before 1925, Dina (Brüser), born in 1923, and Wasser (Tewe) born in 1924. MtDNA haplotypes in the Dina (Geisler) and Dina (Brüser) dam lines, Ho1 and Ho2, respectively, were identified in the previous study of Polish Hovawarts, while a haplotype found in the third line, as yet unknown, was designed as HoU. The bitches represented from the 14th to the 20th descendent generations of the Dina (Geisler) line (hereinafter referred to as the Hol line), the 17 th to the 24th generation of the Dina (Brüser) line (Ho2 line), and 17th generation of the Wasser (Tewe) line ( HoU line). Great differences in the number of representatives of particular lines were found. Most bitches belonged to the Ho2 line, in total 270 individuals, i.e. $73.37 \%$ of the total number of bitches analysed, which, according to the applied methodology, is also the frequency of the Ho2 haplotype, and the least to the HoU line, with only one representative in a kennel from the Nordrhein-Westfalen branch of the RZV. Significant differences among different kennel organizations were found regarding the number of bitches in the lines, and, hence, in the frequency of the haplotypes. The frequency of the dominant $\mathrm{Ho} 2$ haplotype was $84.62 \%$ in $\mathrm{HC}, 81.42 \%$ in RZV, $65.56 \%$ in HZD, and only $41.03 \%$ in SZG.

There were also differences in haplotype frequencies depending on the area of Germany, which is clearly visible on the Fig. 1. In the western part of Germany, which belonged historically to the former Federal Republic of Germany (FRG), the frequency of the Ho2 haplotype was considerably higher than that of the Ho1. The highest Ho2 frequency $(96.3 \%)$ was recorded in the OldenburgNiedersachsen branch of the RZV. In the branches with kennels located in the east, within the former German Democratic Republic (GDR), the frequency of Ho2 was lower; moreover, Hol superiority was noted in two HZD branches - Nord and Ost - at frequencies of 57.1 and $52.2 \%$, respectively. This also applied to kennels of the SZG and HC. In the SZG, the kennels of which are located in the areas of the former East Germany, the Ho1 haplotype $(59.0 \%)$ dominated, while in the HC kennels, located in the former West Germany, Ho2 significant domination $(84.6 \%)$ was observed.

Figure 2 shows the simplified scheme of dam lines and their 75 sublines, including 11 sublines of the Ho1 line and

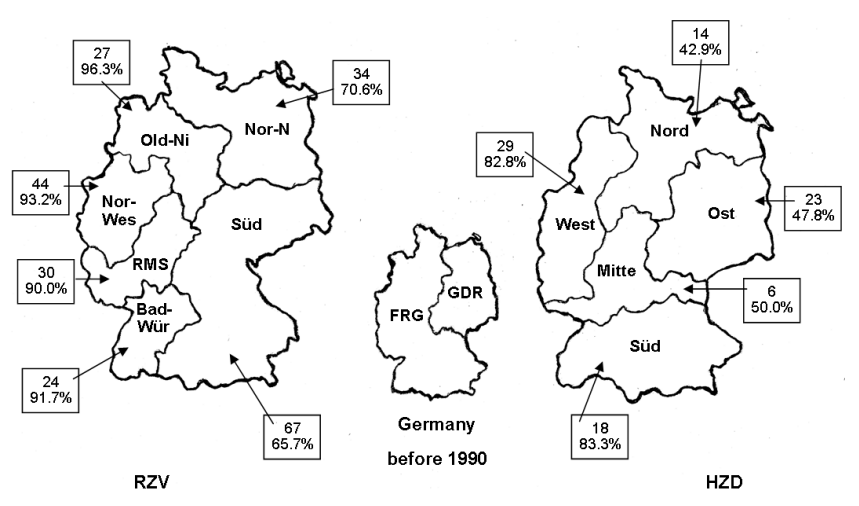

Figure 1. Number of breeding bitches and frequencies of the Ho2 haplotype in the branches ("landesgruppen") of the two German kennel organization: RZV and HZD. Abbreviations: Old$\mathrm{Ni}$ - Oldenburg-Niedersachsen; Nor-N - Nord-Nordost; Nor-Wes - Nordrhein-Westfalen; RMS - Rhein-Main-Saar; Bad-Wür Baden-Württemberg.

63 sublines of the Ho2 line, representing the period from the beginning of the lines to 1989 , i.e. to the period when the Berlin Wall fell and German reunification was initiated. Figure 3 presents the number of modern representatives of the sublines in the different kennel organizations. The different number of sublines in the dam lines and the different number of their representatives is noteworthy here.

Differences among organizations regarding the occurrence of each subline should also be noted. The RZV bitches represented 59 sublines, including 45 of them which are present only in the RZV (hereinafter referred to as the private sublines), while of 21 sublines represented by the HZD bitches, seven are private (Fig. 3). Regarding the other organizations, one and four private sublines were found in the $\mathrm{HC}$ and the SZG, respectively. A noticeably large number of bitches is found in the private subline, labelled subline 6 (Fig. 3); its 14 representatives comprised $35.9 \%$ of all those at SZG. The phenomenon of private sublines also occurs when considering sublines started in more remote generations of ancestors. In the case of 24 sublines derived from the 10th generation, 11 sublines had representatives in RZV kennels exclusively, and 2 sublines in SZG kennels.

The last objective of the study was to evaluate the frequency of potential pedigree errors or mtDNA mutations (EMs) in the modern population of breeding bitches if the source of the EM would be one of the female ancestors in the 5 th (Fig. 4a) or 10th generation of dam lines, respectively (Fig. 4b). The number of ancestors in these chosen generations is limited and equal to 11 ancestors in the 5th generation and 24 ancestors in the 10th generation of the dam lines. The high disproportion in the frequency of the EMs in the modern population resulting from different numbers of subline representatives was found. The lowest frequency occurred in a case of the EM in the HoU line at $0.27 \%$, which was independent of the generation examined in the simula- 




Figure 2. Simplified scheme of three dam lines of German Hovawart dogs including data for the period up to 1989. Names of ancestors of Polish Hovawarts, hair samples of which were used in laboratory mtDNA analysis by Głażewska et al. (2012), are given in rectangles. Right column - subline numbers. 


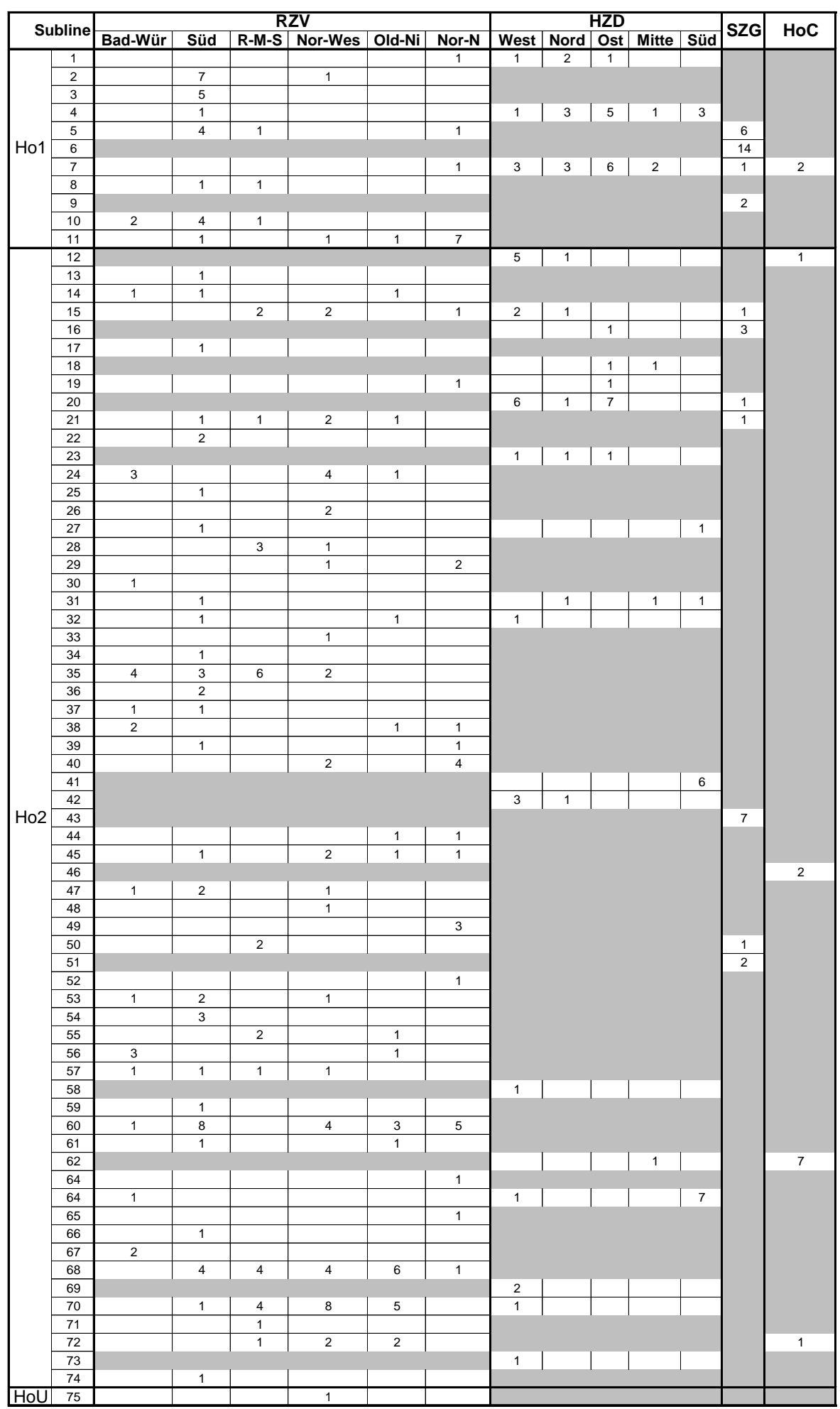

Figure 3. Number of representatives of particular sublines in four kennel organizations: RZV, HZD, SZG and HC, and their branches. Numbers of the sublines correspond to the numbers in the Fig. 2. Abbreviated names of the RZV branches: Old-Ni - Oldenburg-Niedersachsen; Nor-N - Nord-Nordost; Nor-Wes - Nordrhein-Westfalen; RMS - Rhein-Main-Saar; Bad-Wür - Baden-Württemberg. 
A

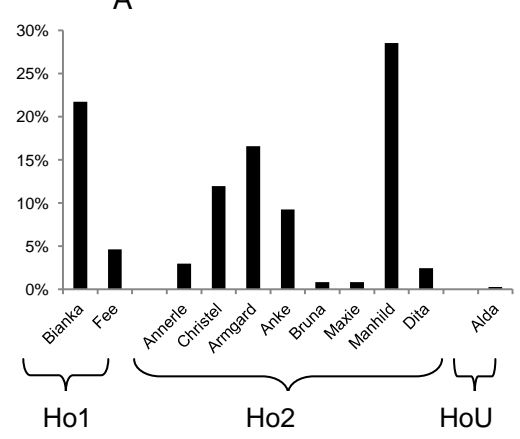

B

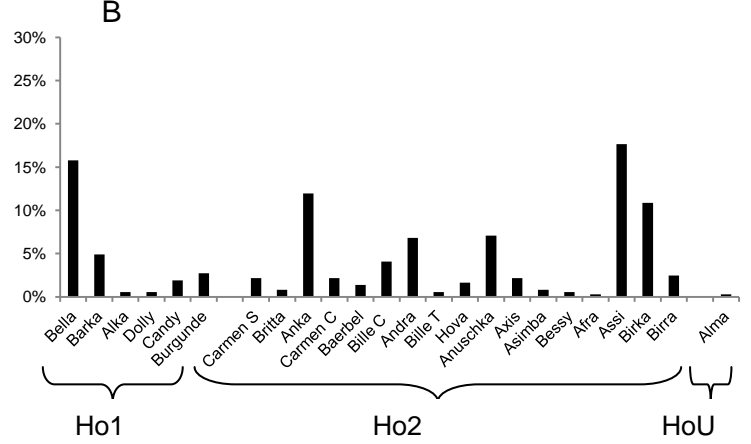

Figure 4. Maximum frequencies of pedigree errors or potential mtDNA point mutations (EMs) in the modern population, assuming that a source of EMs was the female ancestors in the 5th (a) or 10th (b) generation of the lines.

tion because the line has only one representative in the analysed population. Similarly low frequency occur in the case of the EM in the Ho2 line, in the Afra subline originating in the 10th generation (Fig. 4b). In turn, the highest frequencies of the EMs in the 5th generation would be found in the Ho1 line at $21.74 \%$, and in the Ho2 line at $28.53 \%$, while of EMs in the 10th generation, the highest frequencies would be $15.76 \%$ (Ho1) or $17.66 \%$ (Ho2).

\section{Discussion}

The pedigree-based analysis of German Hovawarts provide an interesting picture of mtDNA diversity in dogs. No more than three haplotypes were recognized based on the number of dam lines. Such a low number of mtDNA haplotypes in the relatively large population in the breed's country of origin, in which a rather high level of mtDNA diversity should be present (Savolainen et al., 2002), is quite surprising. The same haplotype number was found in a small population of Polish Hounds (Głażewska and Prusak, 2012), and only one haplotype was found in Karst Shepherds, a local western Balkan breed with a high level of inbreeding and strong founder effect (Ceh and Dovc, 2014). Next, from one to four haplotypes were found in four Portuguese native breeds analysed by Pires et al. (2006), however, more haplotypes were found in two of these breeds: five instead of two haplotypes in the Portuguese Sheepdog and two instead of one in the Castro Laboreiro Dog in the studies by van Asch et al. (2005) performed on a larger number of individuals. Taking into account the above results of the analyses of small local populations, a higher number of mtDNA haplotypes in the Hovawarts was expected.

The low mtDNA diversity in the breed could have been caused by reasons that deserve wider discussion. The first of these could have been the very limited number of breed founders. However, a quick look at the pedigrees of the representatives of the fourth generation of the lines, the names of which are presented in Fig. 2, indicates there are at least 11 female ancestors that can be considered as breed founders.
Unfortunately, dam lines of only three of them are still active in the modern population, and this low number could have resulted from war-time losses.

The second reason could be a closed studbook that precludes introducing bitches of distinct origin with new mtDNA haplotypes into the population. In populations in which only animals already recorded in studbooks are used in breeding, decreases in the number of active dam lines and the losses of their mtDNA haplotypes can be expected over time. This is intensified in dogs by some of the circumstances connected to the specifics of their breeding. Firstly, representatives from only parts of litters are used in breeding. This is evident in Polish Hovawarts at a proportion of $50.7 \%$ of the litters (Głażewska et al., 2012), and similar tendencies can also be expected in German breeding. Secondly, usually only one bitch from a litter is included in breeding (the average in German Hovawarts is 1.15). The one-litter kennels observed in many dog breeds (Leroy et al., 2007; Calboli et al., 2008; Mäki, 2010), which produce disproportionately low numbers of breeding bitches (Głażewska et al., 2012), could also play a role in the random loss of mtDNA haplotypes present in a population. A general rule of "one kennel-one breeding bitch" also facilitates the extinction of dam lines. This was present in the majority of German Hovawarts kennels and observed in the present study, with only 1.1 bitch per kennel, on average. Moreover, kennel breeding time is generally short. This unfavourable trend is apparent in the information on the small percentage of bitches born in the same kennel as their mothers $(38.3 \%)$ and grandmothers $(19.0 \%)$. This problem undoubtedly also concerns other breeds, and generally leads to lower mtDNA diversity in dogs in comparison to that of horses, in which dam lines play an important role in breeding. This concerns such breeds as the Thoroughbred, Lipizzan and Arabian, in populations of which 25, 37 and 74 mtDNA haplotypes, respectively, were found (Bower et al., 2013; Kavar et al., 2002; Khanshour and Cothran, 2013).

In reference to Hovawarts, the question remains open as to whether the haplotype in the Wasser (Tewe) line, here designed as HoU, is identical to the Ho1 or Ho2 haplotype or 
whether it is a third haplotype in the breed, and what the chances are of this line, represented in our analysis by barely one bitch, of surviving. Another interesting question pertains to the number of mtDNA haplotypes in Hovawart populations in other countries that could retain haplotypes originating from other female founders of the breed.

The division of dam lines into many sublines with different number of representatives in the modern population is a typical phenomenon in animal breeding, and the line structure of the population resembles the structure observed in horses. It should be noted that each subline can differ not only with regard to genes in the nuclear DNA, but is also a separate pathway of mtDNA transmission from generation to generation. In the case of an mtDNA mutation fixed only in one subline, the population divides into subpopulations with normal and mutated mtDNA what might be especially important for sampling in genetic studies.

The part of the country in which a particular Hovawart kennel is located and the organization to which it belongs are also very important. The analysis showed strong geographical and historical divisions as exhibited by the dominance of the Ho2 haplotype in West Germany and of the Hol haplotype in the East Germany. The discovery of the persistence of the former political division of Germany, which is visible in differences in haplotype frequencies in breeding bitches from different kennel organizations, is of particular interest. The dominance of the Ho1 haplotype in a group of bitches from the SZG, derived from a GDR kennel organization that has declared its willingness to support the former East German Hovawart (www.szg-hovawart.de/verein), and the strong dominance of $\mathrm{Ho} 2$ in the HC, which brings together kennels only from the former FRG (http://www.hovawart-club.de/zucht-im-hc/ züchter/) and RZV, which was established in 1948 in the FRG (www.hovawart.org), are worthy of special attention. These observations tend to lead to the conclusion that 2 decades after the fall of the Berlin Wall the Iron Curtain still divides. Similar observations concerning the impact of the post-war division of Germany on the genetic structure of a population was also reported by Fritz et al. (2004) in their work on Emys orbicularis turtles. In this case, the object of the study was a wild, randomly mating population, history of which was also influenced by humans and their activity.

The final step of the analysis was to evaluate the scale of potential errors in calculations resulting from de novo mutations and inaccuracies in pedigrees. The frequencies of potential mutations in the simulations ranged widely from 0.27 to $28.53 \%$, and in the case of mutations, which could have occurred in closer ancestral generations, the frequencies were lower correspondingly, up to a single observation of a new mutation in 1 of the 368 bitches studied. However, the numbers above refer to maximum values if all the modern descendants of a given female ancestor from the 5th or 10th generations of the line inherit the mutated mtDNA. In reality, the degree to which a mutation appears in a given line can differ and be complexly embedded in the scheme of family connections. As is demonstrated in studies of humans, a woman with heteroplasmy can transmit a wide range of heteroplasmy to the next generation (Cree et al., 2009). Similar observations were noted in dogs by Klütsch et al. (2011). Heteroplasmy, detected in 3 of 58 pedigrees analysed, occurred only in some representatives of particular family groups in which it was observed, and because of the random transfer of heteroplasmy to the next generation, both mothers and their offspring, as well as individuals from within one litter, differed in the degree of its occurrence.

Potential pedigree errors can occur at the same frequencies as the mutations discussed earlier. However, errors in dam lines seem to be unlikely thanks to the organization of dog breeding, with breeders owning generally only one breeding bitch. On the other hand, such errors cannot be excluded in German Hovawarts, since they have been found in other dog breeds, such as Weimaraners (Kropatsch et al., 2011). Nevertheless, these errors should have relatively small consequences for the quality of our calculations because of the low mtDNA diversity in the population. Erroneous calculations would occur only if a given ancestor with an incorrect pedigree came from another breed or population with a distinct mtDNA haplotype, or from a dam line different than that formally recorded in the pedigree; whereas an error in the same line would obviously not lead to the false identification of a haplotype or an incorrectly calculated haplotype frequencies in the population. Note that the reliability of Hovawart pedigrees was roughly confirmed in the analysis of the Polish population (Głażewska et al., 2012), in which pedigree and mtDNA data exhibited complete agreement (more details are given in Fig. 2). In this context, the results of mtDNA studies in horses, in which pedigree errors are usually identified (Hill et al., 2002; Kavar et al., 2002; Głażewska et al., 2007; Bower et al., 2013), are deserve attention. Of particular interest are the results presented by Hill et al. (2000) and Bower et al. (2013) on Thoroughbreds; these indicate that increased numbers of pedigree errors and haplotypes are identified when more detailed pedigree data are used.

In summary, the present analysis shows that pedigrees can be a valuable source of information in mtDNA studies of pedigreed populations. The results of the study reveal that modern human history, and human decisions and sentiments are important factors influencing genetic diversity and population division of a dog population. Simultaneously, they demonstrate also an interesting paradox: although the differences between the Ho1 and Ho2 haplotypes are the result of events (mutations) from thousands of years ago, the number and the frequencies of the haplotypes in the modern Hovawart population are the result of its history and current breeding policy. This conclusion might be useful also in interpreting results of mtDNA studies of wild species affected by human activity. Finally, pedigree analysis, which allows us to define the inheritance paths of particular mtDNA haplotypes and their mutations, can 
play an important role in scientific studies by increasing efficiency while simultaneously reducing costs.

Edited by: K. Wimmers

Reviewed by: two anonymous referees

\section{References}

Bower, M. A., Whitten, M., Nisbet, R. E. R., Spencer, M., Dominy, K. M., Murphy, A. M., Cassidy, R., Barrett, E., Hill, E. W., and Binns, M.: Thoroughbred racehorse mitochondrial DNA demonstrates closer than expected links between maternal genetic history and pedigree records, J. Anim. Breed. Genet., 130, 227-235, 2013.

Calboli, F. C. F., Sampson, J., Fretwell, N., and Balding, D. J.: Population structure and inbreeding from pedigree analysis of purebred dogs, Genetics, 179, 593-601, 2008.

Ceh, E. and Dovc, P.: Population structure and genetic differentiation of livestock guard dog breeds from the Western Balkans, J. Anim. Breed. Genet., 131, 313-325, 2014.

Cree, L. M., Samuels, D. C., and Chinnery, P. F.: The inheritance of pathogenic mitochondrial DNA mutations, BBA-Mol. Basis Dis., 1792, 1097-1102, 2009.

Fritz, U., Guicking, D., Lenk, P., Joger, U., and Wink, M.: When turtle distribution tells European history: mtDNA haplotypes of Emys orbicularis reflect in Germany former division by the Iron Curtain, Biologia, 59, 19-25, 2004.

Głażewska, I. and Prusak, B.: Evaluation of the effectiveness of introducing new alleles into the gene pool of a rare dog breed: Polish Hound as the example, Czech J. Anim. Sci., 57, 248-254, 2012.

Głażewska, I., Wysocka, A., Gralak, B., Prus, R., and Sell, J.: A new view on dam lines in Polish Arabian horses based on mtDNA analysis, Genet. Sel. Evol., 39, 609-619, 2007.

Głażewska, I., Zielińska, S., and Prusak, B.: Formation of a new dog population observed by pedigree and mtDNA analyses of the Polish Hovawart, Arch. Tierzucht, 55, 391-401, 2012.

Głażewska, I., Prusak, B., and Gralak, B.: Pedigrees as a source of information in mtDNA studies of dogs and horses, Anim. Genet., 44, 227-230, 2013.
Hill, E. W., Bradley, D. G., Al-Barody, M., Ertugrul, O., Splan, R. K., Zakharov, I., and Cunningham, E. P.: History and integrity of thoroughbred dam lines revealed in equine mtDNA variation, Anim. Genet., 33, 287-294, 2002.

Kavar, T., Brem, G., Habe, F., Sölkner, J., and Dovc, P.: History of Lipizzan horse maternal lines as revealed by mtDNA analysis, Genet. Sel. Evol., 34, 635-648, 2002.

Khanshour, A. M. and Cothran, E. G.: Maternal phylogenetic relationships and genetic variation among Arabian horse populations using whole mitochondrial DNA D-loop sequencing, BMC Genet., 14, 83, doi:10.1186/1471-2156-14-83, 2013.

Klütsch, C. F. C., Seppälä, E. H., Uhlen, M., Lohi, H., and Savolainen, P.: Segregation of point mutation heteroplasmy in the control region of dog mtDNA studied systematically in deep generation pedigrees, Int. J. Legal Med., 125, 527-535, 2011.

Kropatsch, R., Streitberger, K., Schulte-Middelmann, T., Dekomien, G., and Epplen, J. T.: On ancestors of dog breeds with focus on Weimaraner hunting dogs, J. Anim. Breed. Genet., 128, 64-72, 2011.

Leroy, G., Verrier, E., Wisner-Bourgeois, C., and Rognon, X.: Breeding goals and breeding practices of French dog breeders: results from a large survey, Rev. Med. Vet.-Toulouse, 158, 496503, 2007.

Mäki, K.: Population structure and genetic diversity of worldwide Nova Scotia Duck Tolling Retriever and Lancashire Heeler dog populations, J. Anim. Breed. Genet., 127, 318-326, 2010.

Pires, A. E., Ouragh, L., Kalboussi, M., Matos, J., Petrucci-Fonseca, F., and Bruford, M. W.: Mitochondrial DNA sequence variation in Portuguese native dog breeds: Diversity and phylogenetic affinities, J. Hered., 97, 318-330, 2006.

Savolainen, P., Zhang, Y. P., Luo, J., Lundeberg, J., and Leitner, T.: Genetic evidence for an East Asian origin of domestic dogs, Science, 298, 1610-1613, 2002.

van Asch, B., Pereira, L., Pereira, F., Santa-Rita, P., Lima, M., and Amorim, A.: MtDNA diversity among four Portuguese autochthonous dog breeds: a fine-scale characterisation, BMC Genet., 6, doi:10.1186/1471-2156-6-37, 2005.

Verscheure, S., Backeljau, T., and Desmyter, S.: Reviewing population studies for forensic purposes: Dog mitochondrial DNA Zookeys, 365, 381-411, doi:10.3897/zookeys.365.5859, 2013. 